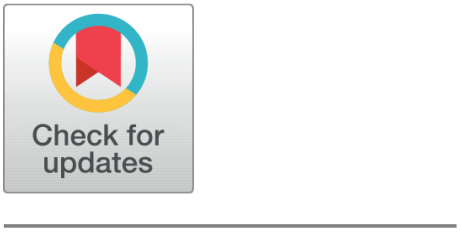

OPEN ACCESS

Received: 08.09.2020

Accepted: 14.07.2021

Published: 27.07 .2021

Citation: Thilagavathy A, Meenakshi S, Vijayabhaskar V, Babu MD, Kumari S, Gunavathie MA (2021) An Efficient Health Monitoring Method Using Fuzzy Inference System via Cloud. Indian Journal of Science and Technology 14(25): 2145-2151. https://doi.org/ 10.17485/IJST/v14i25.1622

* Corresponding author.

atv.cse@rmkec.ac.in

Funding: None

Competing Interests: None

Copyright: (c) 2021 Thilagavathy et al. This is an open access article distributed under the terms of the Creative Commons Attribution License, which permits unrestricted use, distribution, and reproduction in any medium, provided the original author and source are credited.

Published By Indian Society for Education and Environment (iSee)

ISSN

Print: 0974-6846

Electronic: 0974-5645

\section{An Efficient Health Monitoring Method Using Fuzzy Inference System via Cloud}

\author{
A Thilagavathy ${ }^{1 *}, \mathrm{~S}$ Meenakshi ${ }^{2}, \mathrm{~V}$ Vijayabhaskar ${ }^{2}, \mathrm{M} \mathrm{Dilli} \mathrm{Babu}^{3}, \mathrm{~S}$ Kumari $^{3}$, \\ M A Gunavathie ${ }^{3}$
}

1 Department of Computer Science \& Engineering, R.M.K Engineering College, India 2 Department of Science and Humanities, R.M.K Engineering College, India

3 Department of IT, Panimalar Engineering College, Poonamallee, Chennai, Tamil Nadu, India

\section{Abstract}

Background: In the midst of invention of sensor mechanization, massive statistics analytics had turn out to be an innovative prototype for quick information processing. Real-time analytics are required for getting access to the information and its processing. Large populace interest in fitness care led to the improvement of health monitoring systems. Methods: This study targets toward the improvement of a cloud based fitness health care system. Here Wireless Body Area Networks (WBAN) is used to blend the information to the server that includes STORM. The analytics are performed on the received physiological data. The non-critical information is ousted and the essential facts are saved and a notification is sent to the medical doctor. Findings: Thus, actual time analytics alongside with the cloud aid to improve the effectiveness of the proposed healthcare device by providing immediate clinical assistance to the users. A sensible selection making device for approximate reasoning that can manage the uncertainty of imperative threat for human fitness with the usage of fuzzy logic is carried out for discovering the health related problems. Novelty: In this study, an analysis to monitor the fitness hazard which is associated to Blood Pressure, Pulse rate and Kidney function is proposed. Levels of blood stress are analyzed with kidney characteristic by using Glomerular Filtration Rate (GFR). Under this concept, fuzzy logic system is proposed to signify the parameters which can also reason the danger for human fitness and evaluation via the usage of rule base component.

Keywords: Wireless Body Area Networks; health care system; fuzzy logic

\section{Introduction}

Data mining is the vital stride in a procedure referred to as knowledge discovery in database. Numerous lookup areas involving soft computing techniques have added to the discovery of new methods related to data mining applications, more particularly on health care. The sizeable surge in the populace increases with a good-sized utilization of science and technologies has led to a restructuring of the developed metropolis into 
smart living space. Smart living contains quite a few key sides such as governance, infrastructure, technology and healthcare. Here, the cloud based fuzzy logic-controlled healthcare system is proposed. Fast improvement in machine learning techniques inclusive of the Wireless Body Area Networks (WBANs) could restrain the death rate from continual chronic illnesses such as cancers and coronary heart ailment via the screening of the health factors in an actual time environment.

The significance of healthcare systems lies in the indispensable function of statistics science in healthcare systems. The benefits of e-health care systems are: Increased first-rate health services, reduction in cost and proper secure data transfer. The technical infrastructure of pervasive healthcare systems includes Smart Mobile Devices, Wearable Sensors and Smart Environment. Cloud computing involves the usage of available virtualized sources like hardware, software, applications and services. The advantage of cloud computing in health care system is the on-demand provision, pay-per-use model, storing, processing, and handing over patient's critical data, reducing costs in hardware, software and networking. There is vast boom in demand for healthcare offerings due to often prevailing illness in public, increasing older populations, unexpected natural calamities. Concurrently, there are price pressures stemming from the want to do greater and greater fine work with fewer and extra pricey sources and additionally decreased revenue. Expectations for higher outcomes, great therapy and greater price from the healthcare offerings furnished expand the want for point-of-care access to clinical facts and the parallel evolution and adoption of cellular devices, each for clinical team of workers and for patients, are forcing the wait for IT structures to adapt. Also, the big amplify in digitization of clinical documents - along with the accelerating expand in adoption of electronic medical records (EMR), electronic health records (EHR) and personal health records (PHR) - and the growing occurrence of digital outputs from scanning and monitoring devices, such as magnetic resonance imaging (MRI) scanners and bedside video display units and infusers, furnish extra voluminous and diverse digital facts to maximize the possible gain of cloud solutions. Healthcare company structures leveraging cloud-based computing and cloud services provide an array of advantages in assessment to in-house client-server systems; consisting of economic, operational and purposeful advantages.

The financial advantages of cloud computing can be momentous that cloud computing affords fee flexibility and the plausible for decreased costs. Heavy capital expenditure can be avoided, due to the fact IT assets are received on demand as wished and paid for as a working expense. Also, the fee of workforce sources required to install and keep IT sources are blanketed in the fee of cloud computing. Therefore, the want for extra healthcare company professional IT workforce sources and associated expenses may additionally be decreased when the usage of cloud offerings for IaaS and PaaS systems. However, for SaaS options, the cloud provider company takes the major share of responsibility. From an operational perspective, cloud offerings provide scalability and the capacity to alter to demand rapidly. Cloud offerings can provide higher safety and privateness for health records and health systems. Cloud carrier company facts facilities are usually relatively impervious and nicely included towards outsider and insider threats the use of administrative, physical and technical strategies carried out and maintained by way of specialist expert staff. Cloud offerings can provide state-of-the-art safety controls, which includes records encryption and fine-grained get entry to controls and get entry to logging. Medical structures constructed the use of cloud offerings can supply internet get entry to data, fending off the want to save data on purchaser devices.

The want for scarce IT protection competencies inside the healthcare agency additionally is minimized. Cloud provider companies commonly function on such a scale that they have all the critical IT skills, with the charges of these competencies unfold throughout many customers. Arguably, the biggest useful advantage of healthcare cloud offerings is the large vary of new competencies that they are capable to offer. These offerings provide the chance to prolong the competencies reachable to fitness organization staff, in order to put in force higher methods of working and to provide new offerings to patients.

The authors in ${ }^{(1)}$ used IOT which monitors the human fitness with the usage of sensors. The sensor will feel and tune the fitness circumstance and it sends to the storage medium known as cloud computing the place the physician or affected person can capable to see the outcomes every time they want. The Arduino UNO microcontroller is used as a gateway to connect to quite a number sensors. The microcontroller senses the information from the sensor and sends it to the community via Wi-Fi and subsequently gives actual time monitoring of the fitness care parameters for doctors. The records can be accessed each time with the aid of the medical doctor in cloud surroundings storage. The authors in ${ }^{(2)}$ proposed a smart machine to screen affected person's present day fitness conditions, as a smart healthcare machine based totally on the broadly unfold technologies; namely, GSM and GPS to grant a wonderful machine model, that will track, trace, and display affected person fundamental readings in order to supply environment friendly scientific offerings in time. The records will be captured and in contrast with a predefined threshold. The authors in ${ }^{(3)}$ used Wearable sensor mobile technologies and machine learning techniques. They proposed an easy but correct cellular software that is successful of real-time prognosis and monitoring of sufferers with Coronary Artery Disease (CAD) or coronary heart ailment which is a foremost motive of demise worldwide. They constructed a sensible classifier that is successful of predicting a coronary heart disorder hassle primarily based on medical records entered by way of the consumer or the physician and by means of the use of laptop getting to known algorithms. This prognosis element is built-in in the cellular utility with a real-time monitoring factor that continually video display units the affected person and raises an alarm every 
time an emergency occurs. The authors in ${ }^{(4)}$ describe a fuzzy method to PC aided clinical analysis in a scientific context. They brought a formal view of prognosis in medical settings and confirmed the relevance and feasible use of fuzzy cognitive maps and fuzzy logic. A real-time device for detecting the fall of aged humans in smart domestic household was introduced in ${ }^{(5)}$ and $^{(6)}$. The authors in ${ }^{(7)}$ introduced "SESGARH", a scalable and extensible smart-phone primarily based healthcare system, to grant real-time non-stop monitoring of fitness prerequisites of men and women searching for expert healthcare. The authors in ${ }^{(8)}$ developed a superior prediction mannequin so as to estimate the coronary heart rates of chosen sufferers in a mobile care system. The authors in ${ }^{(9)}$ developed a remote machine for monitoring respiratory rate, coronary heart rate and motion behavior of at-home aged human beings who are dwelling alone. The authors in ${ }^{(10)}$ developed an implantable telemetry module for monitoring the heartbeat patient. The authors in ${ }^{(11)}$ developed the machine which used to be centered each for monitoring aged and for monitoring rehabilitation after hospitalization period. The authors in ${ }^{(12)}$ described a novel bed sensing approach for non-invasive, constraint-free and subliminal detection of bio-signals. The authors in ${ }^{(13)}$ applied a domestic self-healthcare monitoring gadget which can display respiration, blood glucose, urinary flow, and temperature. The authors in ${ }^{(14)}$ proposed fitness care monitoring machine that permits vast responsiveness and system optimization through integrating complicated event processing. An approach that involves the usage of the mixture of ZigBee and GPRS is introduced in ${ }^{(15)}$ which is a farflung fitness monitoring device to gather and switch bio-signal facts from the affected person to healthcare center. This device transfers the facts correctly.

The authors in ${ }^{(16)}$ addresses the challenges and proposes a new structure that combines a cloud-based WBANs with statistical modelling strategies in order to offers impervious storage infrastructure and optimize the real-time consumer query processing in phrases of energy minimization. The authors in ${ }^{(17)}$ proposed a convex optimization framework to sketch a communication exchange schedule in the physique sensor community to attain an optimum trade-off between energy consumption and the latency. Their most important notion is to advise a polling based totally algorithm that makes use of time-varying points of person sensors and offers a decision-tree that helps to get to the bottom of scheduling conflicts amongst devices. It estimates the query effects and the sensors solely switch the information if the error is greater than a threshold described via the query. The authors in ${ }^{(18)}$ suggests that the gadget gives an entire unified and cell platform based connectivity answer for unremarkable fitness monitoring. The authors in ${ }^{(19)}$ emphasis the fuzzy logic inter-disciplined with Data Mining that had commented as a pretty novel subject bringing collectively more than a few standards in the network. They had used Fuzzy inference System to derive fuzzy primarily based guidelines for their measurement. The authors in ${ }^{(20)}$ focus on design elements of autonomic cloud surroundings that collect patient's health information and put them into the cloud repository and additionally help evaluation on the records the use of Software offerings hosted in the cloud. There exist numerous recent works ${ }^{(21)}$ to ${ }^{(22)}$ on WBAN related health monitoring systems

\section{Methodology}

In this paper, fuzzy logic system for analyzing hazard for fitness of affected person with respective of the kidney characteristics, blood pressure and pulse rate. The three parameters such as blood pressure, pulse rate and kidney functions are considered because they are the vital basic lifesaving metrics associated with humans. Fuzzy strategies can mark a necessary position in data mining. In our proposed work, we intend to use a low electricity wi-fi, (wireless fidelity), to switch the statistics between the WBAN and the server that allows low power consumption.

The purpose of this paper is to have the funds for essential fitness facts of patients to the physician, caregivers and the hospital administration promptly, furnished if any catastrophic stipulations tracked down. Maneuverability should be achieved by way of acquiring records from these sufferers who are positioned at their house barring any help of external entities such as care takers, medical doctor etc. This may want to be finished through consolidating this information from the WBANs to the subsequent stages for via the internet. At the subsequent layers, the integral attributes are merged and thereby actual time analytics had been performed the usage of the storm, the actual time analyzer with a mixture of the fuzzy logic. If any deviation prevails, then an alert has been raised to emergency services, health facility management, caregivers and the physician. The utility is positioned in a cloud environment to acquire scalability, effectivity and security. The architecture diagram is shown in Figure 1. 


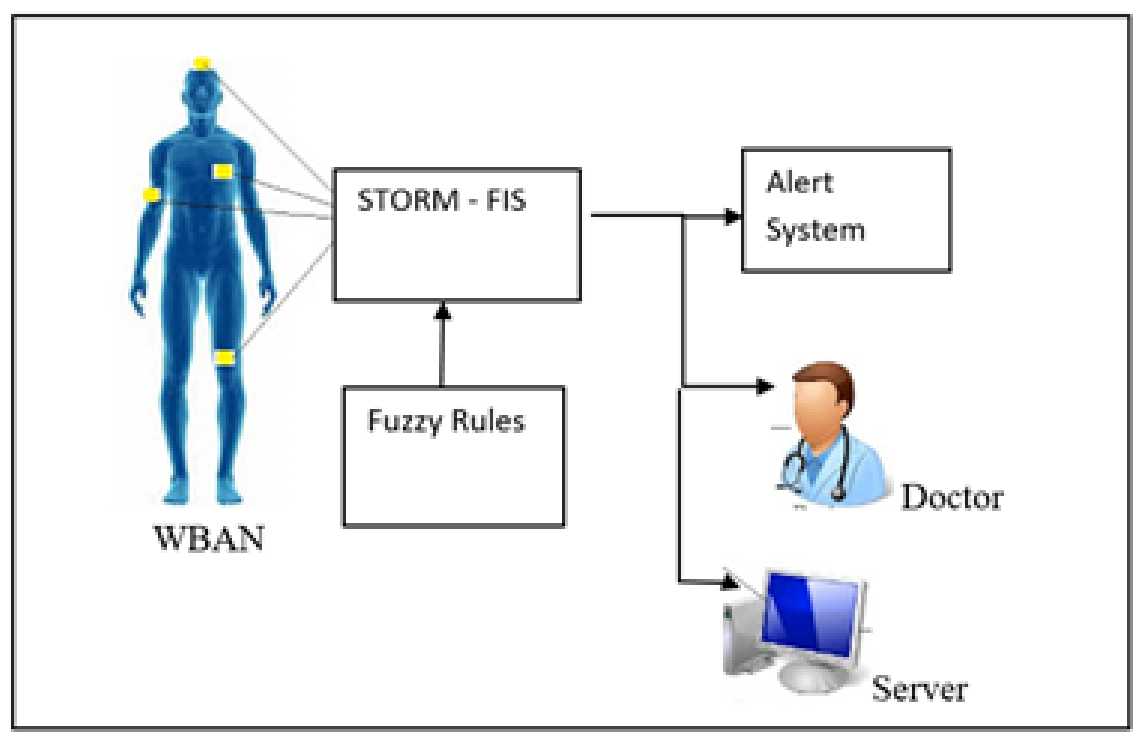

Fig 1. Architechture Diagram

The algorithm involved is explained as follows: From the WBAN, various data are retrieved. The patient is identifies with patient ID. The patient ID is applied with STORM. If that particular patient ID exists, then all the information about that patient is updated in the server. If patient ID is not found, then new registration is done for that patient with a new patient ID. FIS rules is generated for bloop pressure, pulse rate and kidney functions. The health metrics such as low, medium, high is calculated based on the fuzzy sets. The health metrics calculated are sent to the health personnel. If the health metric is found to be very high, then an immediate alert is sent to the doctors and family care keeper of that patient through GPS and immediate attention is given to that patient.

Algorithm:

Input: Data received from WBAN

Output: Health metrics to medical personnel

Step 1: For each patient ID, do

Step 2: Each Patient ID applied with Storm

Step 3: If Patient Id exist in Storm

Update all information about that

patient ID

Else

Perform new registration for

Patient ID

Step 4: Generate FIS rules for BP, pulse rate and Kidney functions

Step 5: Based on fuzzy sets, namely, 'low', 'medium', 'high' and 'very high', health metrics sent to health personnel

Step 6: For 'very high' category, an immediate alert about that patient ID is set using GPS

Step 7: End Process

\section{Implementation and Results}

The fuzzy sets of blood pressure and blood sugar have the triangular membership function as shown in Figure 2. The membership functions are subjected to fuzzy rules as shown in Figure 3. From the MATLAB rule viewer in Figure 4, it can be inferred that with the input blood pressure of 60 and blood sugar of 110, the output shows a risk of 0.5. Similarly, Figure 5 shows that with the input blood pressure of 70 and blood sugar of 110 , the output shows a risk of 0.5. But Figure 6 shows the high risk category of 0.87 when the input blood pressure of 85 and blood sugar of 120 . 


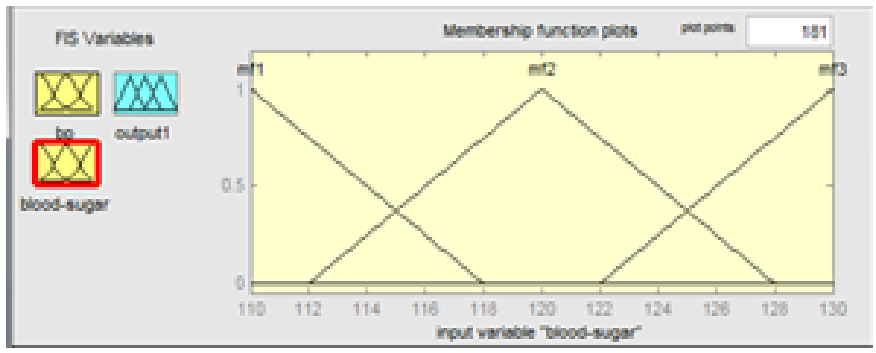

Fig 2. Membership function of blood pressure and blood sugar.

1. If (Cop is $\mathrm{mtr}$ ) and (Blood-sugar is $\mathrm{mt} 1$ ) then (Output 1 is $\mathrm{mt} 1$ ) (1)

3. If (bp is $\mathrm{mt} 1$ ) and (blood-sugar is $\mathrm{mt} 1$ ) then (output 1 is $\mathrm{mr}$ ) (1)

4. If (bop is $\mathrm{mt} 1$ ) and (blood-aupar is $\mathrm{mf} 1$ ) then (outputt is mif) (1)

5. If (bop is $m f t$ ) and (blood-sugar is mf1) then (output1 is mf2) (1)

6. If (op is $\mathrm{mft}$ ) and (blood-sugar is mrt) then (output is mr3) (1)

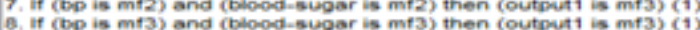

9 . If (bop is mf3) and (blood-sugar is mf3) then (output is mf3) (1)

10. If (Do is mf3) and (biond-sugar is mt3) then (output1 is mf2) (1)

11. If (bp is $\mathrm{mt}$ ) and (blood-sugar is $\mathrm{mt} 1$ ) then (output1 is mf3) (1)

12. If (Dp is $\mathrm{mt}$ ) and (blood-sugar is $\mathrm{mt}$ ) then (output 1 is $\mathrm{mr}$ ) (1)

13. If (bp is $\mathrm{mr}$ ) and (bioded-sugar is $\mathrm{mr}$ ) then (output is $\mathrm{mr}$ ) (1)

14. If (bp is $\mathrm{mr}$ ) and (blood-sugar is $\mathrm{mt}$ ) then (output 1 is $\mathrm{mr} 3$ ) (1)

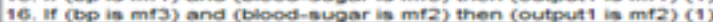

17. If (bp is $\mathrm{mfz}$ ) and (blood-sugar is mf3) then (output1 is mf2) (1)

Fig 3. Fuzzy rules

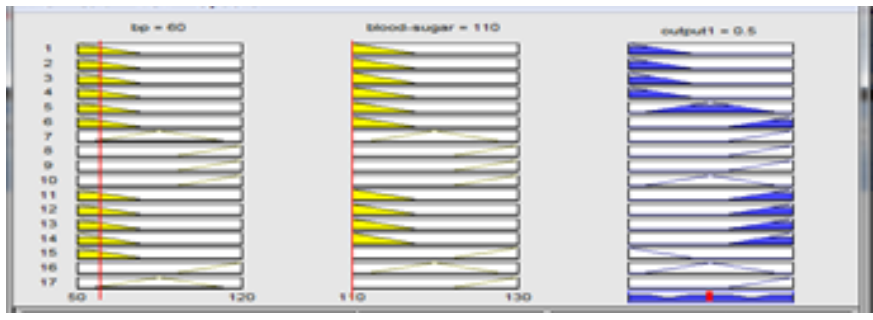

Fig 4. Rule Viewer1

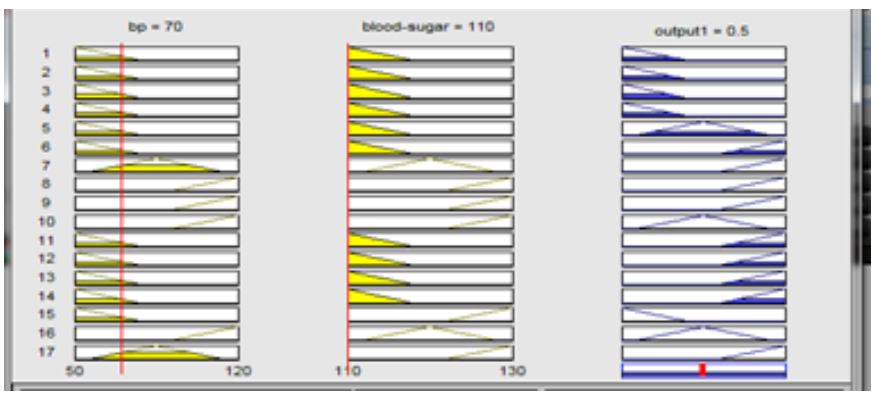

Fig 5. Rule Viewer2 


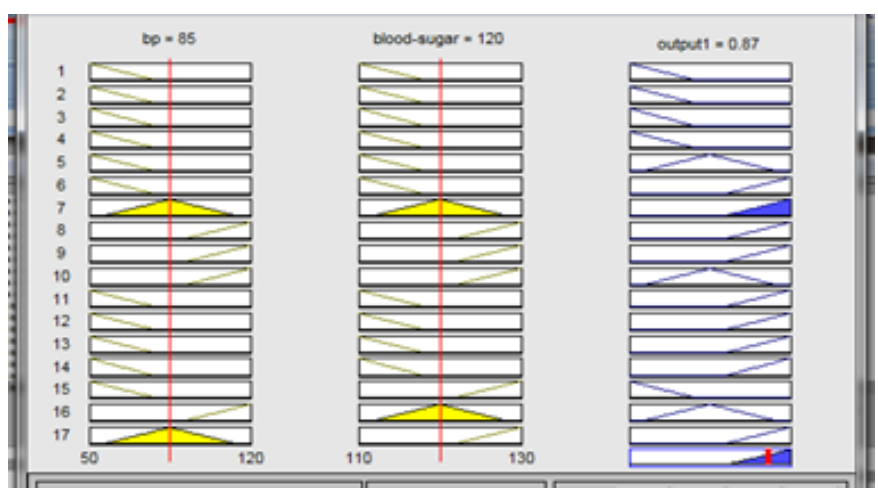

Fig 6. Rule Viewer3

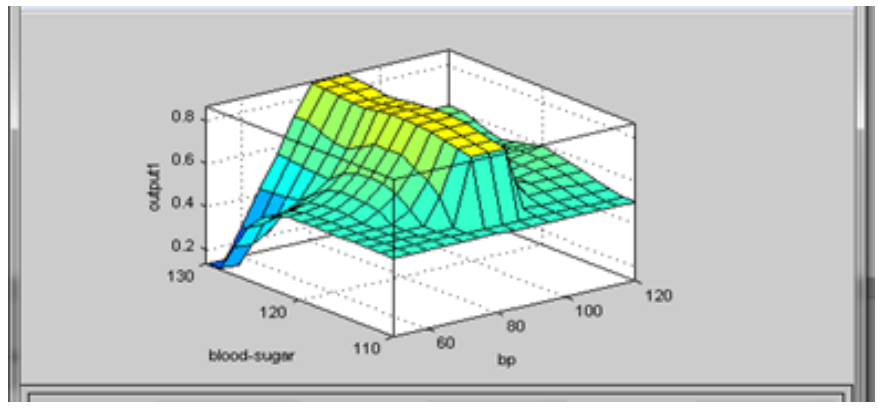

Fig 7. Surface Viewer snapshot

\section{Conclusion}

In this study, Wireless Body Area Networks was used to blend the information to the server that included STORM. The stream analytics are performed on the received physiological data. The non-critical information is ousted and the essential facts are saved and a notification is sent to the medical doctor. Fuzzy logic was carried out in device tool. The fitness hazard which is associated to Blood Pressure, Pulse rate and Kidney function was monitored.

\section{References}

1) Karthigainathan. Human Health Supervision System on Cloud Computing Using Internet of Things". International Journal of Engineering Science and Computing. 2016;6(6).

2) Saed T. Smart Real-Time Healthcare Monitoring and Tracking System using GSM/GPS Technologies". International Journal of Computer Applications. 2016;142(14). Available from: 10.1109/ICBDSC.2016.7460394.

3) Otoom AF, Abdallah EE, Kilani Y, Kefaye A, Ashour M. Effective Diagnosis and Monitoring of Heart Disease. International Journal of Software Engineering and Its Applications. 2015;9(1):143-156. Available from: 10.14257/ijseia.2015.9.1.12.

4) Innocent PR, John RI. Computer aided fuzzy medical diagnosis. Information Sciences. 2004;162(2):81-104. Available from: https://dx.doi.org/10.1016/j. ins.2004.03.003.

5) Poorani D, Ganapathy K, Vaidehi V. Sensor Based Decision Making Inference System for Remote Health Monitoring. IEEE. 2012. Available from: 10.1109/ICRTIT.2012.6206773.

6) Nawka N, Maguliri AK, Sharma D, Saluja P. SESGARH: A Scalable Extensible Smart-Phone based Mobile Gateway and Application for Remote Health Monitoring. IEEE. 2011. Available from: 10.1109/IMSAA.2011.6156341.

7) Khalil I, Su F. Mobile Device Assisted Remote Heart Monitoring and Tachycardia Prediction. In: 5th International Conference on Information Technology and Application in Biomedicine. 2008. doi:10.1109/ITAB.2008.4570646.

8) Mukai K, Yonezawa Y, Ogawa H, Maki H, Caldwell WM. A remote monitor of bed patient cardiac vibration, respiration and movement. In: 31 st Annual International Conference of the IEEE EMBS. 2009. doi:10.1109/IEMBS.2009.5333716.

9) Yutaka H, Yamaguchi H, Kobashil S, Taniguchi K, Nakajima H. A Human Health Monitoring System of Systems in Bed. IEEE. 2008. Available from: 10.1109/SYSOSE.2008.4724147.

10) Kuwana K, Dohi T, Hashimoto Y, Matsumoto K, Shimoyama I. Implantable telemetry capsule for monitoring arterial oxygen saturation and heartbeat. In: 30th Annual International IEEE EMBS Conference Vancouver. 2008. doi:10.1109/IEMBS.2008.4649885.

11) Zakrzewski M, Junnilal S, Vehkaojal A, Kailanto H, Vainio AM, Defee I, et al. Utilization of Wireless Sensor Network for Health Monitoring in Home Environment. IEEE. 2009. Available from: 10.1109/SIES.2009.5196206. 
12) Nukaya S, Shino T, Kurihara Y, Watanabe K, Tanaka H. Noninvasive Bed Sensing of Human Biosignals Via Piezoceramic Devices Sandwiched Between the Floor and Bed. IEEE Sensors Journal. 2012;12(3):431-438. Available from: https://dx.doi.org/10.1109/jsen.2010.2091681.

13) Kim KA, Shin SY, Suh JW, Park C, Cha EJ, Bae HD. Home Healthcare Self-Monitoring System for Chronic Diseases. IEEE International Conference on Consumer Electronics. 2012;p. 486-487. Available from: 10.1109/ICCE.2012.6161987.

14) Vaidehi V, Bhargavi R, Ganapathy K, Hemalatha CS. Multi-sensor Based In-home Health Monitoring using Complex Event Processing. IEEE. 2011. Available from: 10.1109/ICRTIT.2012.6206847.

15) Yu H, Liu L. Remote Health Monitoring System Using ZigBee Network and GPRS Transmission Technology. Fourth International Symposium on Computational Intelligence and Design. 2011. Available from: 10.1109/ISCID.2011.46.

16) Diallo O, Rodrigues J, Sene M, Niu J. Real-Time Query Processing Optimization for Cloud-based Wireless Body Area Networks. Information Sciences. 2014. Available from: 10.1016/j.ins.2014.03.081.

17) Nabar S, Walling J, Poovendran R. Minimizing energy consumption in body sensor networks via convex optimization. In: BSN'10:Proceedings of the2010 International Conference on Body Sensor Networks. IEEE Computer Society. 2010;p. 62-67. doi:10.1109/BSN.2010.19.

18) Shubhangi M, Verulkar, Limkar. Real Time Health Monitoring Using GPRS Technology. International Journal of Computer Science and Network. 2012;1(3). Available from: 10.17148/IJARCCE.2017.61103.

19) Kaur P, Khurmi SS, Josan GS. Fuzzy Based Analysis of Proposed Model for Physical Health Standard Based on Association Rule Mining Techniques. International Journal of Computer Science and Communication Engineering. 2012;1(2).

20) Pandey S, Voorsluys W, Niu S, Khandoker A, Buyya R. An autonomic cloud environment for hosting ECG data analysis services. Future Generation Computer Systems. 2012;28(1):147-154. Available from: https://dx.doi.org/10.1016/j.future.2011.04.022.

21) Kooman JP, Wieringa FP, Han M, Chaudhuri S, van der Sande FM, Usvyat LA, et al. Wearable health devices and personal area networks: can they improve outcomes in haemodialysis patients? Nephrology Dialysis Transplantation. 2020;35(Supplement_2):ii43-ii50. Available from: https://dx.doi.org/10.1093/ ndt/gfaa015.

22) El-Hasnony IM, Barakat SI, Mostafa RR. Optimized ANFIS Model Using Hybrid Metaheuristic Algorithms for Parkinson's Disease Prediction in IoT Environment. IEEE Access. 2020;8:119252-119270. Available from: https://dx.doi.org/10.1109/access.2020.3005614. 